\title{
Magnetic Fano Resonances in All-Dielectric Nanocomplexes under Cylindrical Vector Beams Excitation
}

\author{
Arash Ahmadivand, ${ }^{*}$ Raju Sinha, and Nezih Pala
}

\begin{abstract}
We report on the study of magnetic responses of azimuthally and radially excited all-dielectric symmetric and antisymmetric nanoassemblies. Our studies show that magnetic Fano resonances (FRs) in the optical wavelengths can be excited in silicon nanoparticle assemblies when illuminated by cylindrical vector beams. We present the underlying mechanism by investigating the generation of hot and coldspots in the gap regions between silicon particles. This work proposes promising methods to induce various types of magnetic resonant modes in all-dielectric nanostructures with high efficiency and without breaking the symmetry under the illumination of cylindrical vector beams.
\end{abstract}

KEYWORDS: Azimuthal and radial beams, all-dielectric assemblies, magnetic response, coldspots.

\section{Introduction}

Observation of antisymmetric lineshapes or Fano resonances (FRs) in artificial all-dielectric nanoparticles (NPs) assemblies has received growing interest in the recent years, because of their promising magnetic responses along the visible to the near infrared region (NIR) under the homogenous linear plane wave illumination [1-3]. Possessing extensive applications such as high-order harmonic generation [4,5] and fast switching [6], the efficiently coupled alldielectric NP clusters have been introduced as low-cost and CMOS-compatible structures for fabricating integrated photonic devices. Employing generalized multi-particle Mie theory for assemblies [7], it is shown that high-permittivity NPs under antiparallel electromagnetic (EM) beam excitation support strong magnetic modes with different orders [1,2]. Considering several techniques that have been introduced for inducing FRs for both all-dielectric and metallic clusters, recently, a new type of FRs is excited in the metallic planar trimer based on its magnetic response, called magnetic Fano resonances (MFRs), which is correlating with the 
formation of strong magnetic hotspot in the offset gaps [8,9]. Newly, using the inherent superior magnetic response of all-dielectric NPs, multiple MFRs were reported in the antisymmetric quadrumer antennas [10]. In comparison to the plasmonic structures, to arise a magnetic dipolar mode in the all-dielectric systems phase retardation is not required between the geometry of the structure and the excited EM modes. However, inducing energetic magnetic hotspots was achieved with the expense of breaking the symmetry of the structures, which requires for highly complex analytical computations and number of fabrication processes. For the symmetric structures, in the previous studies, conventional FRs have already been observed for plasmonic seven-member heptamer nanoassembly, while its symmetry was perturbed [11,12]. Observation of the analogous optical responses were not reported for alldielectric symmetric nanocomplexes. It should be underlined that all of the reported experiments and analysis regarding monitoring different types of FRs and MFRs in all-dielectric assemblies were accomplished for the nanoantennas under linearly polarized beam exposure.

On the other hand, it is shown that metallic NPs assemblies under inhomogeneous cylindrical vector beams (CVBs) excitations show unique behavior and excitation of eigenmodes that cannot be observed in the linear regime $[13,14]$. The growing interest to the CVB polarizations originates from tighten focusing spot size [15], as well as superior conforming between the polarization of the interacting beam and geometrical symmetry of the nanoantenna [16]. Using CVB illumination technique, therefore, arising of ultrastrong hotspots would be possible due to the intensification of the excited magnetic modes.

In this context, by adopting a successful concept from plasmonic studies, we analyze the spectral response for an all-dielectric symmetric heptamer consisting of silicon (Si) NPs under CVB polarizations. Considering the distinct FR dips in plasmonic structures under linear beam exposure, and no FR for the CVB illumination, we investigate the possibility of inducing MFRs in all-dielectric heptamers under CBV excitation. Our numerical analysis verify the formation of strong magnetic hotspots across the visible band, under the azimuthal polarized (AP) beam, while for the radial polarization (RP) opposite results are observed.

\section{Theory and methods}

We used Si NPs with the diameter and height of $170 \mathrm{~nm}$ and $120 \mathrm{~nm}$, respectively, with the offset gap of $20 \mathrm{~nm}$, and empirically measured optical constants taken for Si NPs and substrate [16]. In general, understanding of FRs supported by a multiparticle assembly needs for analyzing the interference between magnetic and electric modes in a nanosystem. Dipole- 
dipole interaction is one of the promising approaches for these calculations, however, this method cannot be fully employed for CVB illumination approach $[17,18]$. Therefore, to analyze the spectral features based on multiparticle Mie theory, the expansion form of all of the incident electric and excited magnetic fields ( $\mathrm{H}$-fields) in terms of vector spherical harmonics must be included in computations to apply the workspace region enclosed by perfectly matched layer (PML) boundaries $[19,20]$. Hence, for a cluster composed of "N" NPs, the spherical harmonics for the total EM excited fields including both scattered electric and internal $\mathrm{H}$-fields corresponding to the $n$th particle can be described by $[21,22]$ :

$$
\begin{aligned}
& E_{s c a t}^{n}\left(r_{n}\right)=\sum_{i=1}^{\infty} \sum_{k=-l}^{l} A_{k l}^{n} \frac{?_{k l 3}^{?}}{?}\left(r_{n}\right)+B_{k l}^{n} H_{k l 3}^{n}\left(r_{n}\right) \\
& H_{\mathrm{int}}^{n}\left(r_{n}\right)=\sum_{l=1}^{\infty} \sum_{k=-l}^{l} C_{k l}^{n} \prod_{k l 1}^{?}\left(r_{n}\right)+D_{k l}^{n} H_{k l 1}^{n} ?
\end{aligned}
$$

where $J$ and $H$ are the spherical harmonics according to the Gérardy and Ausloos definitions [20]. The numbers that are used as indices in the equations above and below $(1,3)$ refer to the spherical harmonics correlating with the Bessel function [21]. Moreover, the externally applied electric and magnetic dipolar moments are discussed in the literature comprehensively [2,23]. Besides, the scattered magnetic fields $\left(H_{s c a t}\right)$ can be described using vectorial spherical harmonics as follows:

$$
H_{\mathrm{scat}}^{n}\left(r_{n}\right)=\sum_{i=1}^{\infty} \sum_{k=-l}^{l} E_{k l}^{n} ?_{k l 3}^{?}\left(r_{n}\right)+F_{k l}^{n} H_{k l 3}^{n} ?
$$

where

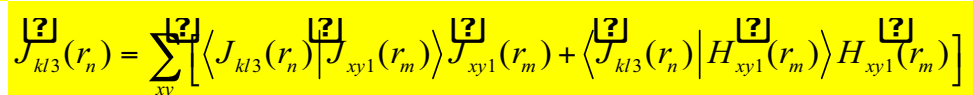

$$
\begin{aligned}
& \left.\stackrel{?}{H_{k l 3}}\left(r_{n}\right)=\sum_{x y}^{\text {? }}\left\langle H_{k l 3}\left(r_{n}\right) J_{x y 1}\left(r_{m}\right)\right\rangle ?_{x y 1}^{\text {? }}\left(r_{m}\right)+\left\langle\stackrel{?}{H}_{k l 3}\left(r_{n}\right) \mid H_{x y 1}^{?}\left(r_{m}\right)\right\rangle H_{x y 1}\left(r_{m}\right)\right]
\end{aligned}
$$

and the definitions for the harmonics are listed here:

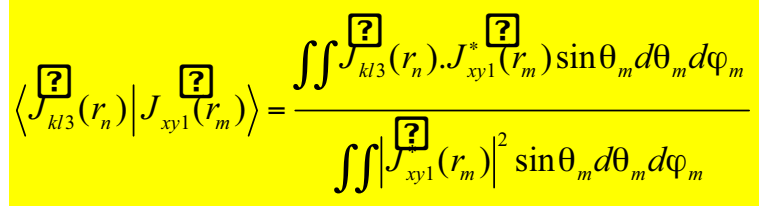

In addition, the incident magnetic beam $\left(H_{\text {inc }}\right)$ can be described by:

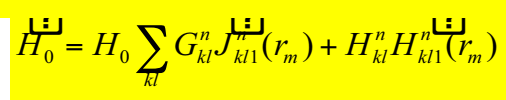


Considering the provided spherical coefficients and functions for $m$ spherical NPs, the Maxwell equations for the overall matrix can be rewritten as follows consisting of $H_{0}$ and diffracted magnetic field $\left(H_{M}=H_{0}+H_{D}\right)$ :

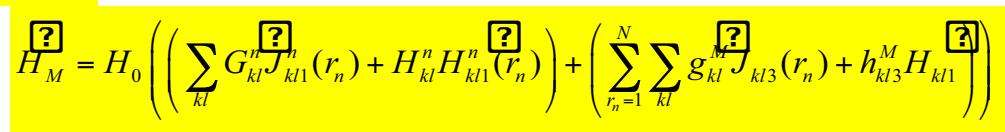

To plot the extinguished scattering spectra, the scattered power can be used as a function of the incident beam. One should note that the conventional scattering and extinction crosssections cannot be used for CVB illuminations, therefore, the scattered power by integration of Poynting vectors $[13,21,23]$. Therefore, the scattered field for the incident cylindrical plane wave is given by [21]:

$$
P_{s c a t}=\frac{1}{2 Z k^{2}} \sum_{n, k, l}\left(\left|E_{k l}^{n}\right|^{2}+\left|F_{k l}^{n}\right|^{2}+\operatorname{Re}\left(\left(\frac{E_{k l}^{n}}{\Gamma_{k}^{n}}-G_{k l}^{n}\right) E_{k l}^{n^{*}}+\left(\frac{F_{k l}^{n}}{\Delta_{k}^{n}}-H_{k l}^{n}\right) F_{k l}^{n^{*}}\right)\right)
$$

where, $Z$ is the workplace impedance, and $1 / \Delta$ and $1 / \Gamma$ are the diagonal matrices formed by the inverse of the magnetic susceptibilities [21]. It is well understood that for an all-dielectric heptamer cluster with the perturbed symmetry under the linear beam excitation, a distinct dip as a FR can be induced in the proximity of the middle NP which supports strong magnetic dipolar mode [1,2]. It should be noted that the oscillating direction of the optically induced magnetic moments in the central and peripheral NPs are opposite (out-of-phase regime). However, the same strategy cannot be employed in CVBs polarizations, where for both symmetric and antisymmetric plasmonic heptamer systems, no significant magnetic and electric moments observed in the central NP, while the peripheral NPs are able to sustain significant dipolar and multipolar moments [14].

All numerical analysis were performed employing commercial Lumerical FDTD Solutions software. We assumed that the entire workspace is surrounded with perfectly matched layers (PMLs) with the number of 16 absorptive layers to prevent any destructive interference between the cylindrically excited magnetic resonances and scattered ones in the workplace. The bandwidths of the CVB sources were set to $500 \mathrm{~nm}$ to $1200 \mathrm{~nm}$. The grid sizes for all of the axes are set to $0.5 \mathrm{~nm}$. Also, for the theoretical explanations and to characterize the physical mechanism behind, we utilized generalized Mie theory for cluster-type structures.

\section{Results and discussions}


Figures 1a and 1b illustrate the perspective images for the all-dielectric nanoantenna on a $\mathrm{Si} / \mathrm{SiO}_{2}$ surface for both AP and RP illuminations. The initially obtained results (the extinguished power profiles) for the all-dielectric antenna under AP and RP illuminations are plotted in Fig. 1c and 1d, respectively, which are correlating with the extinction spectra in the homogenously excited systems. For the AP light, we observed a noticeable minimum along the plotted curve at $735 \mathrm{~nm}$, arising due to the out-of-phase condition between the excited modes. The underlying physics behind the formation of the MFR mode (iii) could be explained as follows. Noticing in the corresponding $\mathrm{H}$-field map (see Fig. 1e), the central particle is almost neutralized in terms of dipolar and multipolar moments, while the surrounding NPs reflect very strong magnetic dipolar and multipolar resonances. This condition implies that the induced $\mathrm{H}$ fields held by NPs are partially out-of-phase and in-phase over each radiation steps, therefore, both types of opposite modes can be excited in an individual NP. Herein, the field generated by each NP opposes the induced dipolar momentum of the neighbor one, hence, we expected a significant field enhancement and overlap between surrounding dielectric particles for the AP radiation. Qualitatively, the induced EM fields in an individual NP amplify the dipolar moment of the proximal NP, leading to a remarkably larger field enhancement. In addition, the extremes appeared around the FR dip are correlating with the magnetic response of the peripheral NPs. The narrower peak (i) at shorter spectra $(650 \mathrm{~nm})$ is associating with the multipolar magnetic mode and the broader one (ii) in the longer spectra $(820 \mathrm{~nm})$ is relating with the dipolar magnetic mode. During the rotation of the polarized light around the rotationally symmetric nanoassembly, in the out-of-phase regime, a destructive interference between dipolar and multipolar resonant peaks of adjacent particles results in formation of an antisymmetric lineshape in between (MFR dip). The other important spectral feature is the formation of a giant and localized coldspot centering in the middle part of the cluster, which can be employed for high resolution spectroscopic, subwavelength florescence, and imaging purposes [24].

In contrast, for the RP light, the coupling efficiency between NPs is poor due to the weak field enhancement in the in-phase regime. Here, the excited charges repelling each other in the outward direction. In the extinguished power profile (Fig. 1d), the broader dipolar magnetic peak became narrower, while the multipolar peak is damped drastically. Therefore, in the inphase limit, the MFR minimum disappeared. The corresponding charge density distribution also shows the direction of charges and low energy of the excited magnetic resonant modes inside the NPs (see Fig. 1f). Comparing both RP and AP lights, for the AP beam, an admixture of the excited energetic dipolar magnetic modes and multipolar ones led to formation of a coil- 
type resonance along the visible band. Also, in terms of quality of the coldspots, for the RP beam, these coldspots are weak. To show the unique behavior of the all-dielectric antenna under CVB beams, we plotted the $\mathrm{H}$-field enhancement $\left(|H| /\left|H_{0}\right|\right)$ profile for the beams with different polarizations along with the extinguished power curve in Fig. 2a. The distinguished magnetic field enhancement for the AP beam exposure due to the strongly localized field enhancement in the cluster at the position of MFRs (solid line, around $\lambda \sim 760 \mathrm{~nm}$ ) is clearly visible. The blue-shift and damping of the field enhancement are indicated by a dotted line and an arrow for the RP beam (dashed line). For the latest polarized beam, the enhancement is dramatically reduced due to losing the confinement of the magnetic modes. In addition, to indicate the magnetic response of the structure, we compared currently examined all-dielectric cluster with the recently reported works by Nazir et al. [9] and Ahmadivand et al. [10] in Fig. 2b. A quick look at the profile, we realize a significant enhancement of the $\mathrm{H}$-field in the symmetric heptamer cluster at the position of Fano dip under AP beam illumination. On the other hand, for the antisymmetric plasmonic trimer and all-dielectric quadrumer, the $\mathrm{H}$-field localization is lower in the linear regime. This difference in the field enhancement exclusivity originates from the complexity of the cluster, magnetic resonance support of Si NPs, and finally, the type of incident polarization beam which helps to localize the $\mathrm{H}$-field in a tiny spot.

Plasmonic response of metallic nanostructure strongly depends on the gap spots and geometrical features. In contrast, spectral response of all-dielectric clusters is not sensitive to geometrical perturbations. All these dependencies were studied for the linear regime, while for the CVB illuminations, breaking the symmetry can result in with different responses. To reveal this effect, in continue, we analyze the effect of symmetry breaking on the optical spectrum of the all-dielectric cluster under CVBs excitations. Due to negligible effect of the middle NP in our analysis, we majorly focused on the effect of peripheral NPs, and also, the results for AP beam illumination are shown. Figure 3a exhibits top-view profiles for the heptamer antenna, while one of the surrounding NPs is going to be smaller than the others, gradually. Keeping the diameter of all NPs fixed at $170 \mathrm{~nm}$, and reducing the diameter of the indicated NP to $100 \mathrm{~nm}$, the extinguished power spectra for the $\mathrm{H}$-field is illustrated in Fig. 3b. It should be noted that the effect of variations in the offset gap between NPs is negligible. However, during illumination, the energy of the dipolar and multipolar magnetic modes that are oscillating in smaller NP is different than the ones in the larger NPs. This perturbation in the geometry of the cluster leads to a significant red-shift in the position of the MFR signature, while it becomes broader. Broadening (for a disk with the diameter of $160 \mathrm{~nm}$ and $130 \mathrm{~nm}$ ) and disappearing 
(for a disk with the diameter of $100 \mathrm{~nm}$ and NP removed regimes) of the MFR are originated by the incomplete coupling of the rotational EM field flowing through the peripheral NPs, as well as destructive interference of the rotationally excited $\mathrm{H}$-fields between them. For the modified disk with the diameter of $160 \mathrm{~nm}$ and $130 \mathrm{~nm}$, the dipolar magnetic momentum can be efficiently coupled to the nearest particle magnetic modes, while the multipolar mode does not contain enough energy to able be to interact with the nearby NP's H-fields. Hence, the required overlap between dipolar and multipolar modes cannot be accomplished efficiently, resulting with a broader and weaker MFR. In addition, magnetic peaks are damped drastically during the geometrical variations. Further decrements in the size of the Si nanodisk led to spectrally redshift in the position of magnetic modes and FR mode including an increasing broadening in the lineshape of FR dip. As a specific case, for the absence of NP, the optical response of the cluster becomes anisotropic due to the severe perturbations in the symmetry. Figure $3 c$ shows the $\mathrm{H}$-field map for the magnetic resonance excitation for the variations in the size of specific NP under AP beam excitation. The noteworthy point here is the formation of distinguished coldspots as hotspots become weaker, which are more distinct in comparison to entirely symmetric analysis. This can be better understood by plotting the far-field maps for the magnetic field excitation across the all-dielectric nanocluster in different symmetric and antisymmetric regimes, as shown in Fig. 4. As expected for the symmetric regime, the required $\mathrm{H}$-field enhancement is observed across the nanoantenna, while for the antisymmetric clusters, the magnetic field is disturbed and the required $\mathrm{H}$-field confinement is not accomplished effectively. Figure 4(a) shows this effect for a full symmetric heptamer, while Figs. 4(b) and 4(c) are for the case of antisymmetric regimes with the changing diameter of variant particle.

\section{Conclusions}

In conclusion, we have studied the spectral response of both symmetric and antisymmetric all-dielectric molecular clusters under tightly focused AP and RP beams illuminations. We have observed formation of strong magnetic MFR across the visible spectra via overlapping of the excited dipolar and multipolar magnetic charges with different phases, including significantly enhanced $\mathrm{H}$-field, as shown in the near-field profiles. Comparing the localized magnetic field due to CVBs with analogous and antisymmetric metallic clusters (in linear regime), the superior magnetic response of the proposed all-dielectric antenna is verified. In addition, we have shown that the illumination method allows us observing significantly pronounced coldspots in the central parts of clusters, which are comparable with plasmonically induced coldspots in 
both size and quality. The achieved results in the presented work can be employed in designing highly efficient and low-cost and compact devices such as routers, accurate sensors, and high resolution imagers.

\section{Acknowledgements}

This work is supported by NSF CAREER program with the Award number: 0955013, and by Army Research Laboratory ( $A R L)$ Multiscale Multidisciplinary Modeling of Electronic Materials (MSME) Collaborative Research Alliance (CRA) (Grant No. W911NF-12-2-0023, Program Manager: Dr. Meredith L. Reed). Raju Sinha gratefully acknowledges the financial support provided through dissertation year fellowship by the University Graduate School (UGS) at Florida International University.

\section{References}

1.A. E. Miroshnichenko, and Y. S. Kivshar, Fano resonances in all-dielectric oligomers, Nano Lett. 12, 6459 (2012).

2.K. E. Chong, B. Hopkins, I. Staude, A. E. Miroshnichenko, J. Dominguez, M. Decker, D. N. Neshev, I. Brener, and Y. S. Kivshar, Observation of Fano resonances in all-dielectric nanoparticle oligomers, Small 10, 1985 (2014).

3.D. S. Filonov, A. P. Slobozhanyuk, A. E. Krasnok, P. A. Belov, E. A. Nenasheva, B. Hopkins, A. E. Miroshnichenko, and Y. S. Kivshar, Near-field mapping of Fano resonances in all-dielectric oligomers, Appl. Phys. Lett. 104, 021104 (2014).

4.M. R. Shcherbakov, D. N. Neshev, B. Hopkins, A. S. Shorokhov, I. Staude, E. V. Melik-Gaykazyan, M. Decker, A. A. Ezhov, A. E. Miroshnichenko, I. Brener, A. A. Fedyanin, and Y. S. Kivshar, Enhanced third-harmonic generation in silicon nanoparticles driven by magnetic response, Nano Lett. 14, 6488 (2014).

5.M. R. Shcherbakov, A. S. Shorokhov, D. N. Neshev, B. Hopkins, I. Staude, E. V. Melik-Gaykazyan, A. A. Ezhov, A. E. Miroshnichenko, I. Berner, A. A. Fedyanin, and Y. S. Kivshar, Nonlinear interference and tailorable third-harmonic generation from dielectric oligomers, ACS Photonics 2, 578 (2015).

6.A. Ahmadivand, S. Golmohammadi, M. Karabiyik, and N. Pala, Self-assembled silicon-based clusters to design efficient, fast, and controllable Fano switches, Microw. Opt. Technol. Lett. 57, 1242 (2015).

7.Y. -I. Xu, Electromagnetic scattering by an aggregate of spheres, Appl. Opt. 34, 4573 (1995). 
8.A. Nazir, S. Panaro, R. P. Zaccaria, C. Liberale, F. De Angelis, and A. Toma, Fano coil-type resonance for magnetic hot-spot generation, Nano Lett. 14, 3166 (2014).

9.A. E. Miroshnichenko, S. Flach, and Y. S. Kivshar, Fano resonances in nanoscale structures, Rev. Mod. Phys. 82, 2257 (2010).

10.A. Ahmadivand, and N. Pala, Multiple coil-type Fano resonances in all-dielectric antisymmetric quadrumers, Opt. Quant. Electron. 47, 2055 (2015).

11.B. Luk'yanchuk, N. I. Zheludev, S. A. Maier, N. J. Halas, P. Nordlander, H. Giessen, C. T. Chong, The Fano resonance in plasmonic nanostructures and metamaterials, Nat. Mater. 9, 707 (2010).

12.J. A. Fan, C. Wu, K. Bao, J. Bao, R. Bardhan, N. J. Halas, V. N. Manoharan, P. Nordlander, G. Shvets, and F. Capasso, Self-assembled plasmonic nanoparticle clusters, Science 328, 1135 (2010).

13.J. Sancho-Parramon, and S. Bosch, Dark modes and Fano resonances in plasmonic clusters excited by cylindrical vector beams, ACS Nano 6, 8415 (2012).

14.A. Yanai, M. Grajower, G. M. Lerman, M. Hentschel, H. Giessen, and U. Levy, Near- and far-field properties of plasmonic oligomers under radially and azimuthally polarized light excitation, ACS Nano 8, 4969 (2014).

15.W. Chen, and Q. Zhan, Realization of an evanescent Bessel beam via surface plasmon interference excited by a radially polarized beam, Opt. Lett. 34, 722 (2009).

16.E. D. Palik, Handbook of optical constants of solids (Academic Press, CA, USA, 1998).

17.B. Hopkins, A. N. Poddubny, A. E. Miroshnichenko, and Y. S. Kivshar, Revisiting the physics of Fano resonances for nanoparticle oligomers, Phys. Rev. A 88, 053819 (2013).

18.P. Albella, M. A. Poyli, M. K. Schmidt, S. A. Maier, F. Moreno, J. J. Saenz, and J. Aizpurua, Low-loss electric and magnetic field-enhanced spectroscopy with subwavelength silicon dimers, J. Phys. Chem. C 117, 13573-13584 (2013).

19.T. X. Hoang, X. Chen, and C. J. R. Sheppard, Multiple theory for tight focusing of polarized light, including radially polarized and other special cases, J. Opt. Soc. Am. A 29, 32 (2012).

20.R. Borghi, M. Santarsiero, M. A. Alonso, Highly focused spirally polarized beams, J. Opt. Soc. Am. A 22, 1420 (2005).

21.J. M. Gérardy, and M. Ausloos, Absorption spectrum of clusters of spheres from the general solution of Maxwell's equations II. Optical properties of aggregated metal spheres, Phys. Rev. B, 25, 4204 (1982).

22.D. W. Mackowski, Calculation of total cross-sections of multiple-sphere clusters, J. Opt. Soc. Am. A, 11, 2851-2861 (1994). 
23.M. Haggui, M. Dridi, J. Plain, S. Marguet, H. Perez, G. C. Schatz, G. P. Wiederrecht, S. K. Gray, and R. Bachelot, Spatial confinement of electromagnetic hot and cold spots in gold nanocubes, ACS Nano, 6, 1299 (2012).

24.B. Hopkins, D. S. Filonov, A. E. Miroshnichenko, F. Monticone, A. Alú, and Y. S. Kivshar, Interplay of magnetic responses in all-dielectric oligomers to realize magnetic Fano resonances, ACS Photonics, 2, 724 (2015). 

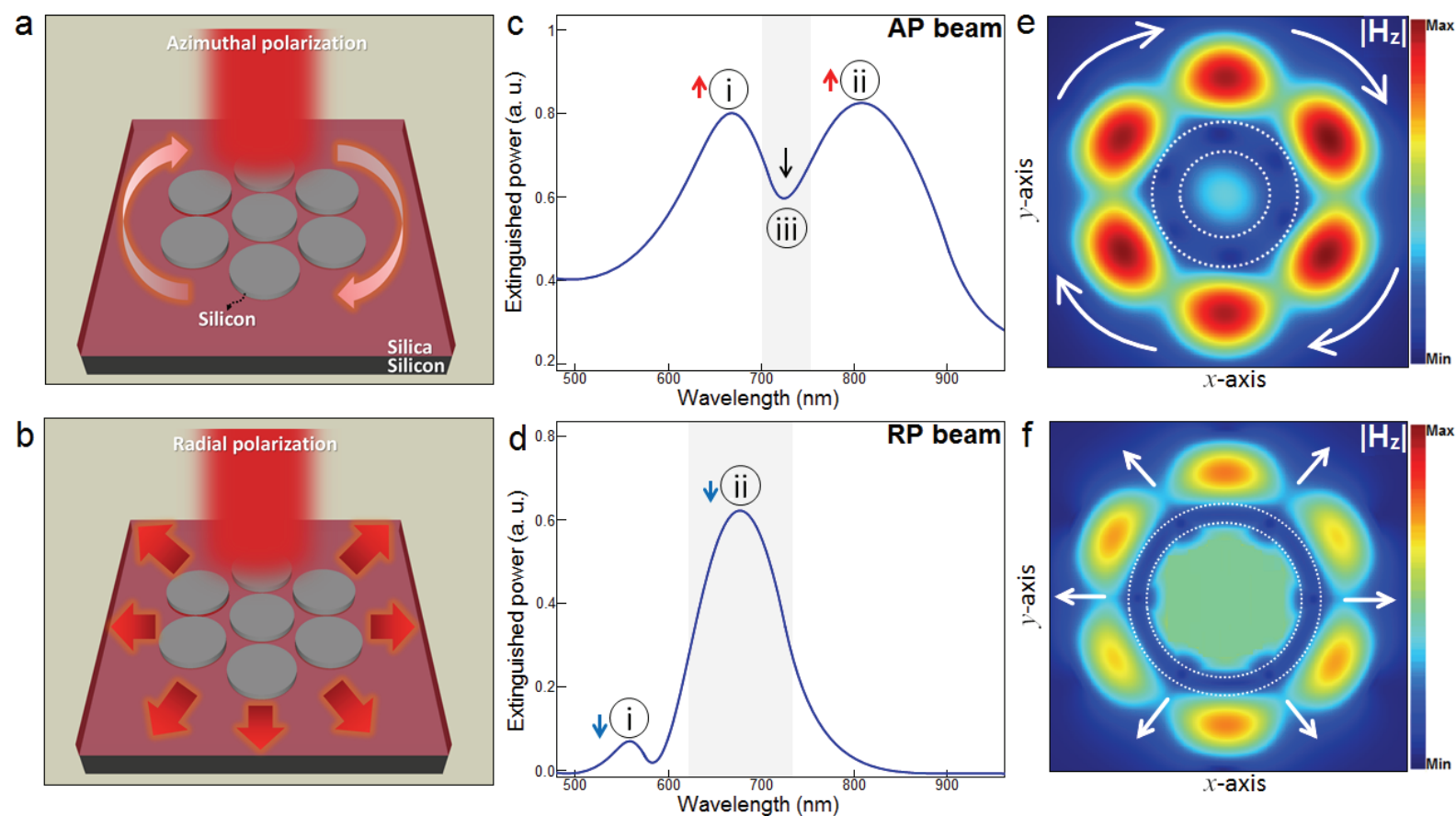

Fig. 1. (a), (b) Schematic perspectives for the all-dielectric heptamer antenna under AP and RP beams, respectively. (c), (d) Extinguished power spectra for the all-dielectric symmetric cluster under AP and RP illuminations, respectively. The arrows show the excitation and damping of the magnetic modes extremes. The spectral features are specified for the quadrupolar (i) and dipolar (ii) magnetic modes, and FCTR mode (iii). (e), (f) Near-field maps for the H-fields $\left(\left|H_{z}\right|\right)$ excitation and confinement in the NPs, under AP and RP beams, respectively. The circular regions indicated by dotted curves corresponding to the coldspot region. The arrows show the charge density motion direction for both polarized cylindrical beams. 

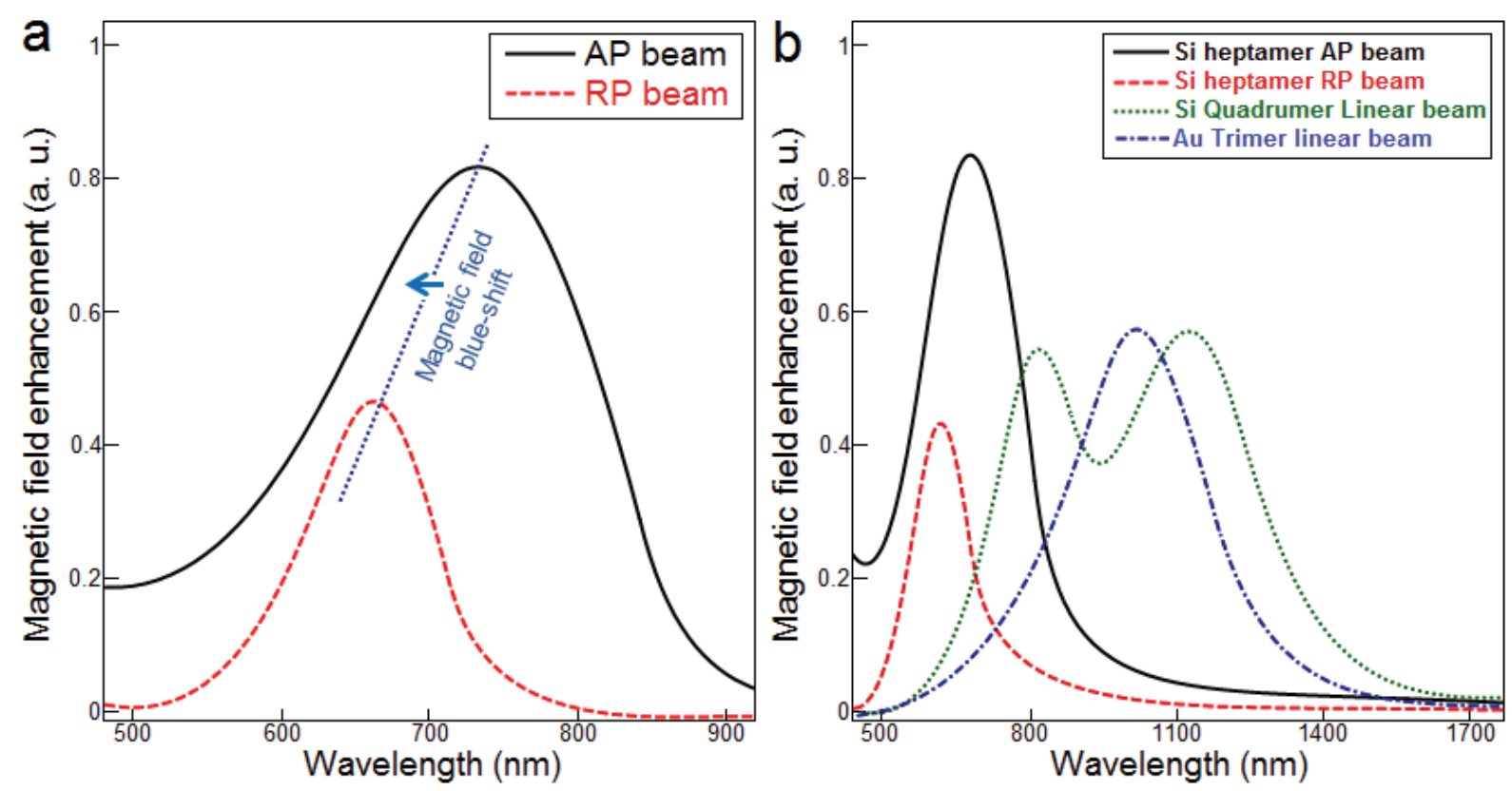

Fig. 2. (a) H-field enhancement $\left(|H||| H_{0} \mid\right)$ for the all-dielectric cluster under AP and RP beams excitations. (b) A comparison between $\mathrm{H}$-field enhancements between different types of all-dielectric and metallic symmetric and antisymmetric nanoscale assemblies. 
a

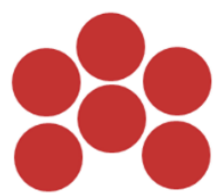

NP Removed
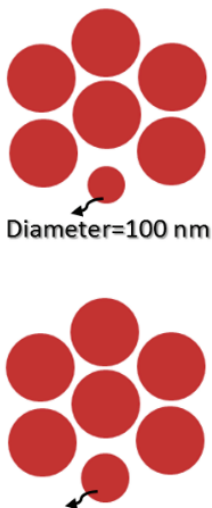

Diameter $=130 \mathrm{~nm}$

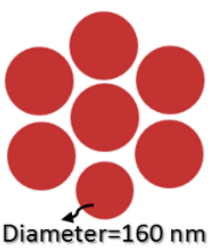

b

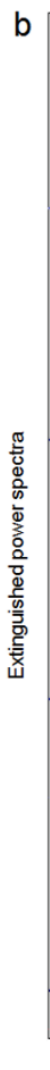

C

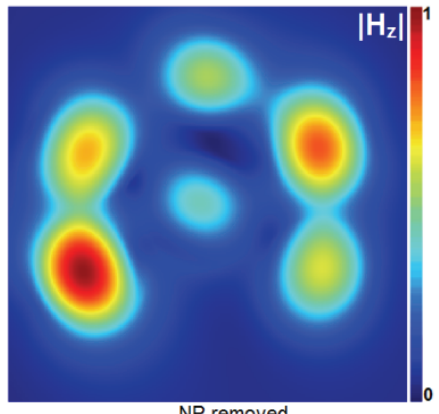

$\mathrm{NP}$ removed

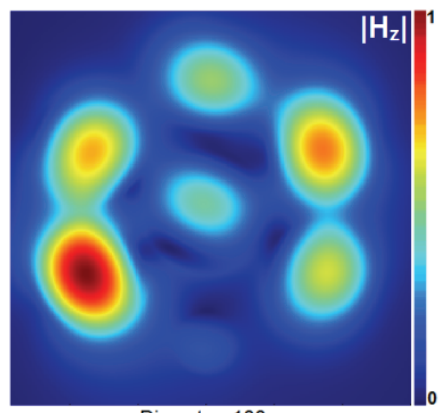

Diameter $=100 \mathrm{~nm}$

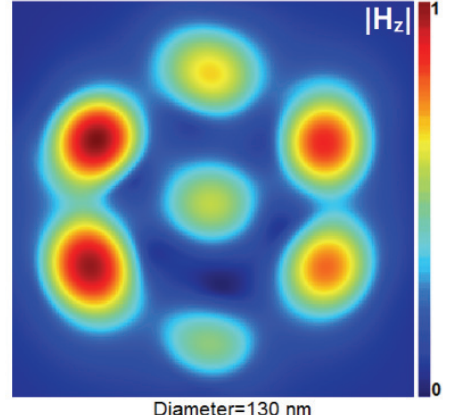

Diameter $=130 \mathrm{~nm}$

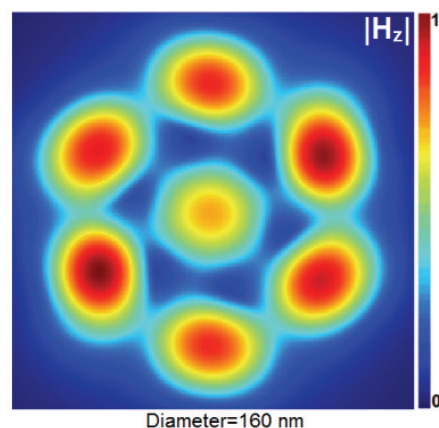

Fig. 3. (a) Top-view profiles for the all-dielectric heptamer, while one of the Si NPs is gradually became smaller. (b) The extinguished power spectra as a function of source bandwidth for the antisymmetric heptamer under AP beam exposure. The diameter of the indicated NP reducing from $160 \mathrm{~nm}$ until NP is removed. The FCTR dip is disappeared for the antisymmetric assembly. (c) The $\mathrm{H}$-field maps $\left(\left|H_{z}\right|\right)$ for the antisymmetric all-dielectric nanoantenna under AP beam exposure, while the size of one of NPs is reducing, and dramatic weakness in the energy of magnetic resonances is obvious. 

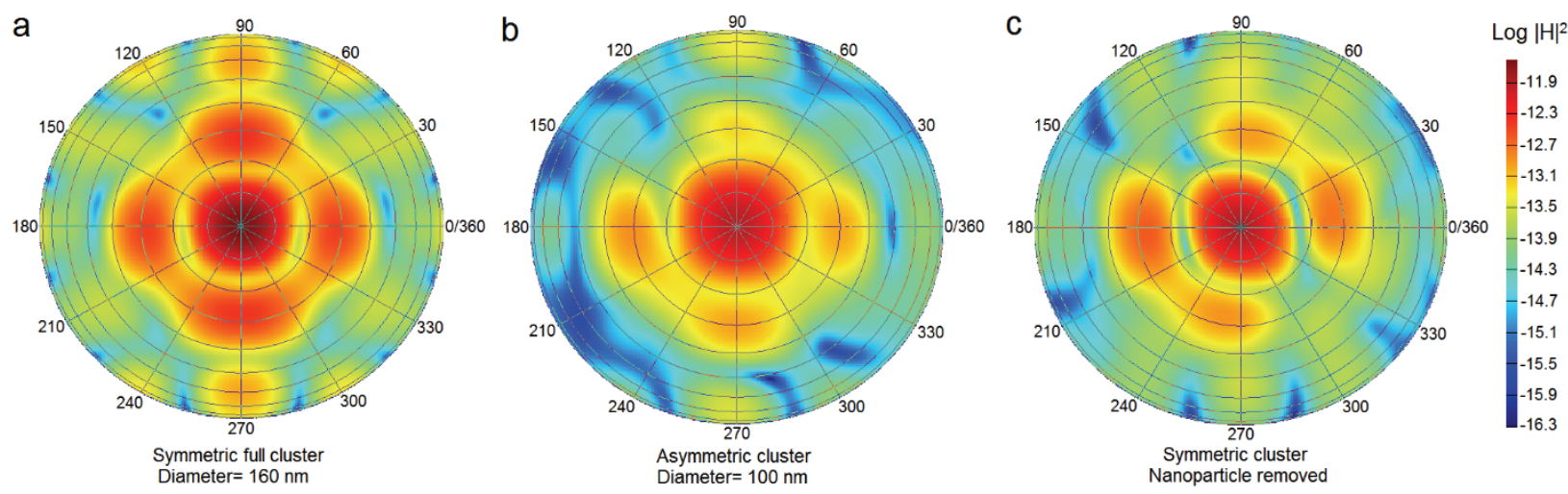

Fig. 4. The magnetic far-field spectrum for the all-dielectric nanoassembly. Far-field map for the symmetric heptamer, with the diameter of the variant particle is a) $160 \mathrm{~nm}$, b) $100 \mathrm{~nm}$, and c) particle is removed. 